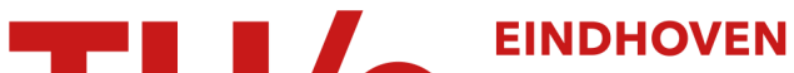 \\ UNIVERSITY OF \\ TECHNOLOGY
}

\section{Phase coexistence and line tension in ternary lipid systems}

\section{Citation for published version (APA):}

Idema, T., Leeuwen, van, J. M. J., \& Storm, C. (2009). Phase coexistence and line tension in ternary lipid systems. Physical Review E - Statistical, Nonlinear, and Soft Matter Physics, 80(4), 041924-1/9. [041924]. https://doi.org/10.1103/PhysRevE.80.041924

DOI:

10.1103/PhysRevE.80.041924

Document status and date:

Published: 01/01/2009

\section{Document Version:}

Publisher's PDF, also known as Version of Record (includes final page, issue and volume numbers)

\section{Please check the document version of this publication:}

- A submitted manuscript is the version of the article upon submission and before peer-review. There can be important differences between the submitted version and the official published version of record. People interested in the research are advised to contact the author for the final version of the publication, or visit the $\mathrm{DOI}$ to the publisher's website.

- The final author version and the galley proof are versions of the publication after peer review.

- The final published version features the final layout of the paper including the volume, issue and page numbers.

Link to publication

\section{General rights}

Copyright and moral rights for the publications made accessible in the public portal are retained by the authors and/or other copyright owners and it is a condition of accessing publications that users recognise and abide by the legal requirements associated with these rights.

- Users may download and print one copy of any publication from the public portal for the purpose of private study or research.

- You may not further distribute the material or use it for any profit-making activity or commercial gain

- You may freely distribute the URL identifying the publication in the public portal.

If the publication is distributed under the terms of Article 25fa of the Dutch Copyright Act, indicated by the "Taverne" license above, please follow below link for the End User Agreement:

www.tue.nl/taverne

Take down policy

If you believe that this document breaches copyright please contact us at:

openaccess@tue.nl

providing details and we will investigate your claim. 


\title{
Phase coexistence and line tension in ternary lipid systems
}

\author{
T. Idema, ${ }^{1}$ J. M. J. van Leeuwen, ${ }^{1}$ and C. Storm ${ }^{1,2}$ \\ ${ }_{1}^{1}$ Instituut-Lorentz, for Theoretical Physics, Leiden Institute of Physics, Leiden University, P.O. Box 9506, 2300 RA Leiden, \\ The Netherlands \\ ${ }^{2}$ Department of Applied Physics and Institute for Complex Molecular Systems, Eindhoven University of Technology, \\ P.O. Box 513, 5600 MB Eindhoven, The Netherlands
}

(Received 17 July 2009; revised manuscript received 8 September 2009; published 23 October 2009)

\begin{abstract}
The ternary system consisting of cholesterol, a saturated lipid, and an unsaturated one exhibits a rich phase behavior with multiple phase coexistence regimes. Remarkably, phase separation even occurs when each of the three binary systems consisting of two of these components is a uniform mixture. We use a Flory-Huggins like model in which the phase separation of the ternary system is a consequence of an interaction between all three components to describe the system. From the associated Gibbs free energy we calculate phase diagrams, spinodals, and critical points. Moreover, we use a Van der Waals/Cahn-Hilliard like construction to derive an expression for the line tension between coexisting phases. We show how the line tension depends on the position in the phase diagram, and give an explicit expression for the concentration profile at the phase boundary.
\end{abstract}

DOI: 10.1103/PhysRevE.80.041924

PACS number(s): 87.16.D-, 87.15.Zg

\section{INTRODUCTION}

Motivated by the possibility of the existence of functional "rafts" in the plasma membrane of living cells [1], many groups have studied artificial or reconstituted biomimetic lipid membranes in recent years [2-5]. Biomimetic membrane vesicles are a good model system for the biological membrane and moreover constitute an interesting soft matter system in their own right. One of the key findings is that simple membranes containing a binary or ternary mixture of lipids can phase separate into coexisting domains under general conditions [6-12]. A typical ternary model system consists of a high melting temperature lipid with saturated tails ("saturated lipid"), a low melting temperature lipid with unsaturated tails ("unsaturated lipid"), and cholesterol. Below the melting temperature the lipids organize in a gel phase, above it they form a liquid phase. There are several possible liquid phases, which are characterized by the long-range orientational order of the tails of the various lipids in the membrane. Saturated lipids, possibly in a mixture with cholesterol, form a liquid phase which is known as liquid-ordered $\left(L_{\mathrm{o}}\right)$, whereas unsaturated lipids form a liquid-disordered $\left(L_{\mathrm{d}}\right)$ phase [13]. The coexisting domains found in a phaseseparated ternary lipid membrane can be a liquid and a gel phase, but also two liquid phases and sometimes even three different phases simultaneously.

A key characteristic of heterogeneity in a lipid membrane is the emergence of a line tension on the boundary between coexisting domains. This line tension plays an important role in determining the overall membrane shape [8,14-17]. In recent years, several groups have sought to determine the line tension in experiment, especially in vesicles exhibiting coexistence of a $L_{\mathrm{o}}$ and a $L_{\mathrm{d}}$ phase [16,18-20]. Moreover, several attempts have been made to calculate the line tension from a microscopical model $[15,21,22]$. In this paper, we use a model for the Gibbs free energy of ternary lipid systems from which we can calculate the line tension in a straightforward manner using a Van der Waals/Cahn-Hilliard like con- struction. This approach relates the measured phase diagrams and line tensions in ternary systems, and gives a prediction on how the line tension will vary due to a change in membrane composition.

The phase behavior of a ternary lipid system depends on the pressure, temperature, and exact membrane composition. For an introduction into the properties of the phase diagrams of these systems see Veatch and Keller [10]. A slice through the phase diagram at constant pressure and temperature can be represented as a Gibbs phase triangle. In such a triangle the vertices represent lipid membranes of uniform composition, the edges binary mixtures and the interior ternary ones. The maximum number of phases $P$ that can coexist in a given system is determined by the Gibbs phase rule [23]

$$
P=C-F+2 \text {. }
$$

Here $C$ is the number of components and $F$ denotes the number of degrees of freedom, i.e., the number of intensive variables which are independent of other intensive variables. In our ternary system $(C=3)$ we have $F=2$ (temperature and pressure) and hence $P=3$. In a binary system only two coexisting phases are possible. Both are consistent with observations, and in recent years several experimentally determined Gibbs phase triangles for ternary lipid systems have been published, showing two- and three-phase coexistence regions $[10,11]$. Remarkably, there are also ternary systems for which each of the three limit binary systems is completely mixed, but for which the ternary system shows a two-phase coexistence region [24]. Since phase coexistence is understood to be a consequence of what is known as a miscibility gap (the effect that the free energy can be lowered by demixing), such phase diagrams are said to contain a closed-loop miscibility gap.

The proper free energy to describe a Gibbs phase triangle is the Gibbs free energy, which has the temperature, pressure and number of particles of each of the components as parameters. For a related system, the mixing of two polymers, Flory-Huggins theory gives an expression of the change in 
free energy due to mixing (see e.g., [25]), consisting of two contributions: an increase in entropy due to the increase in number of possible configurations (which favors mixing) and a term which represents the interactions between individual polymers and is characterized by the Flory-Huggins parameter $\chi$. For positive values of $\chi$ the interaction term favors demixing. If both polymers are equal in size, we have

$$
\mathcal{G}=N k_{\mathrm{B}} T(x \log x+y \log y+\chi x y)
$$

where $x$ and $y$ are the number fractions of the two polymers and $N$ is the total number of polymers.

In a 2004 paper, Komura et al. [26] combined a FloryHuggins like approach for liquid-liquid phase coexistence with an order-parameter description for the liquid-gel phase transition. They presented phase diagrams for two of the three limiting binary systems of the ternary system considered here. In a follow-up paper in 2005 [27] the authors extended their model to the ternary system, introducing three independent Flory-Huggins parameters for the three binary interactions and keeping the order-parameter description for the gel phase. This model allows for a qualitative description of some of the experimentally observed phase diagrams, but fails to reproduce the one with the closed-loop miscibility gap. In an alternative approach, Radhakrishnan and McConnell $[28,29]$ proposed a model in which two of the three components form a complex which interacts with the third component. The resulting phase diagram has some qualitative features which also appear in the closed-loop experimental one of Veatch et al. [24], but does not allow for three coexisting phases. Recently Putzel and Schick [30] presented a refined version of the model of Komura et al. In their work two different models are used, one for the system with a closed-loop miscibility gap and one for the system with a three-phase coexistence region, both depending on a combination of a Flory-Huggins model and an order-parameter description. Using these models, Putzel and Schick also studied the effect cross-linking molecules have on the phase diagram [31].

In this paper we use a model for the ternary system based solely on an extension of the Flory-Huggins model of the binary system, and reducing to the binary models in each of the limit cases. In this model, we supplement the binary interactions with an interaction between all three components. This approach to model a ternary system is well known in the fields of alloys and of polymer mixtures [32-36], but thus far has not been applied to lipid mixtures. We show that the extension with a ternary term is necessary to explain the phase triangle with a closed-loop miscibility gap found experimentally by Veatch et al. [24] when the binary interactions are repulsive. The model can also reproduce the phase triangle with coexisting liquid and gel phases, as well as a three-phase coexistence region. We use our model to determine the linear stability of the system and explicitly find the critical points. Using the expression for the Gibbs free energy given by our model, we can calculate the energy associated with a boundary between two coexisting phases [37]. This boundary energy is a line tension in two-dimensional lipid membranes.
In Sec. II we review the thermodynamics of mixtures, and in Sec. III we discuss the properties of the model we use to describe ternary mixtures. The main result of this paper, the calculation of the line tension as a function of membrane composition, is given in Sec. IV.

\section{THERMODYNAMICS OF MIXTURES}

The appropriate characteristic function for describing phase equilibria in mixtures is the Gibbs free energy, which is a function of the particle numbers $N_{i}$, pressure $p$, and temperature $T$

$$
\mathcal{G}=\mathcal{G}\left(N_{1}, \ldots, N_{n}, p, T\right) .
$$

The requirement for two phases to coexist is that all chemical potentials are equal in both phases, as well as the temperature and pressure (which is why $\mathcal{G}$ is such a useful function for mixtures). The chemical potentials associated with each of the components are given by:

$$
\mu_{i}=\frac{\partial \mathcal{G}}{\partial N_{i}},
$$

where the partial derivatives are taken with all the other variables constant. The total number of particles $N=N_{1}+\cdots$ $+N_{n}$ is constant and taken as the extensive variable, and we define

$$
\mathcal{G} / N=g\left(x_{1}, \ldots, x_{n}\right) \quad \text { with } \quad x_{i}=N_{i} / N .
$$

The number fractions $x_{i}$ have a redundancy, and obey the condition

$$
x_{1}+\cdots+x_{n}=1,
$$

which will allow us to eliminate one of them below. We can write the chemical potentials explicitly as functions of $g$ and its derivatives to the $x_{i}$ 's, showing that they are intensive

$$
\mu_{i}=g+\frac{\partial g}{\partial x_{i}}-\sum_{j=1}^{n} x_{j} \frac{\partial g}{\partial x_{j}} .
$$

These derivatives are unrestricted, in the sense that only the other particle numbers $N_{k}$ are kept fixed, not, e.g., the total particle number $N$. Summing all the $\mu_{i}$ 's, we find that we also have the relation

$$
g=\sum_{i=1}^{n} x_{i} \mu_{i}
$$

Because our system is restricted to the subspace defined by Eq. (6), we can eliminate one of the number fractions (which we take to be $x_{n}$ ) from the problem. Within this subspace, Eq. (7) reads

$$
\begin{gathered}
\mu_{i}=g+\frac{\partial g}{\partial x_{i}}-\sum_{j=1}^{n-1} x_{j} \frac{\partial g}{\partial x_{j}} \quad i=1, \ldots, n-1 \\
\mu_{n}=g-\sum_{j=1}^{n-1} x_{j} \frac{\partial g}{\partial x_{j}}
\end{gathered}
$$

where $g$ and its derivatives are now functions of $x_{1}, \ldots, x_{n-1}$. 
The formalism given above applies to a system with any number of components. For simplicity we will restrict ourselves to ternary systems below. We will indicate the concentrations of the three components by $x, y$, and $z$ instead of $x_{1}$, $x_{2}$, and $x_{3}$. In order to have phase coexistence the chemical potentials of all three components must be equal in both phases. In our ternary system we find that phases with number fractions $\left(\bar{x}_{1}, \bar{y}_{1}\right)$ and $\left(\bar{x}_{2}, \bar{y}_{2}\right)$ can coexist if

$$
\begin{aligned}
& \mu_{1}\left(\bar{x}_{1}, \bar{y}_{1}\right)=\mu_{1}\left(\bar{x}_{2}, \bar{y}_{2}\right), \\
& \mu_{2}\left(\bar{x}_{1}, \bar{y}_{1}\right)=\mu_{2}\left(\bar{x}_{2}, \bar{y}_{2}\right), \\
& \mu_{3}\left(\bar{x}_{1}, \bar{y}_{1}\right)=\mu_{3}\left(\bar{x}_{2}, \bar{y}_{2}\right) .
\end{aligned}
$$

The system Eq. (11) gives us three equations for the four unknowns $\left(\bar{x}_{1}, \bar{y}_{1}, \bar{x}_{2}, \bar{y}_{2}\right)$, which means that in the Gibbs phase triangle there can be a coexistence region, in accordance with the Gibbs phase rule Eq. (1). The boundary of the phase coexistence regime [which consists of pairs of points that satisfy Eq. (11)] is called the binodal.

Using the identities Eqs. (9) and (10), we find that there is an equivalent system of conditions for phase coexistence given by

$$
\begin{gathered}
g_{x}\left(\bar{x}_{1}, \bar{y}_{1}\right)=g_{x}\left(\bar{x}_{2}, \bar{y}_{2}\right), \\
g_{y}\left(\bar{x}_{1}, \bar{y}_{1}\right)=g_{y}\left(\bar{x}_{2}, \bar{y}_{2}\right), \\
g\left(\bar{x}_{1}, \bar{y}_{1}\right)-\bar{x}_{1} g_{x}\left(\bar{x}_{1}, \bar{y}_{1}\right)-\bar{y}_{1} g_{y}\left(\bar{x}_{1}, \bar{y}_{1}\right) \\
=g\left(\bar{x}_{2}, \bar{y}_{2}\right)-\bar{x}_{2} g_{x}\left(\bar{x}_{2}, \bar{y}_{2}\right)-\bar{y}_{2} g_{y}\left(\bar{x}_{2}, \bar{y}_{2}\right),
\end{gathered}
$$

where subscripts $x$ and $y$ on $g(x, y)$ denote derivatives with respect to $x$ and $y$. The first equation of Eq. (12) is found by subtracting $\mu_{3}$ from $\mu_{1}$, the second by subtracting $\mu_{3}$ from $\mu_{2}$ and the third is identical to the third of Eq. (11).

The binodal separates the region in the phase diagrams in which our system is in a homogeneous phase from those in which it separates into two or three coexisting phases. However, in this simple Van der Waals type of phase coexistence, the appearance of an unstable regime in the Gibbs phase triangle is a prerequisite. We therefore study the linear stability of our system at such a point $(x, y)$ in a ternary system. We can vary both number fractions independently, and find for the variation in Gibbs free energy per particle

$$
\delta g=\frac{1}{2}(\delta x, \delta y)\left(\begin{array}{ll}
g_{x x} & g_{x y} \\
g_{x y} & g_{y y}
\end{array}\right)\left(\begin{array}{l}
\delta x \\
\delta y
\end{array}\right)+O(3)
$$

where $O(3)$ refers to third-order terms in $\delta x$ and $\delta y$. For the second-order term in Eq. (13) to vanish the determinant of the matrix $\left(g_{i j}\right)$ of second-order derivatives of $g$ must be equal to zero. This condition also holds for systems with more than three components, and in general we find that the system becomes linearly unstable when

$$
\operatorname{det}\left(g_{i j}\right)=0 \text {. }
$$

We call the set of solutions of Eq. (14) the spinodal, because it marks the boundary between two types of demixing. Linearly stable systems demix by nucleation and growth and linearly unstable ones by spinodal decomposition [25,38]. They are qualitatively different: in the case of nucleation and growth there is a nucleation barrier for the system to overcome before phase separation can take place, which is absent in the case of spinodal decomposition. Binary polymer systems, described by similar two-component Flory-Huggins models, also exhibit distinctly different patterning in the binodal (nucleated) and spinodal regimes [25].

Equation (14) is equivalent with the condition that $\left(g_{i j}\right)$ must have a zero eigenvalue, and if Eq. (14) holds the eigenvalue equation

$$
\sum_{j=1}^{2} g_{i j} r_{j}=0
$$

has a solution in spinodal points. The eigenvector $\vec{r}=\left(r_{1}, r_{2}\right)$, belonging to the eigenvalue 0 , is a direction in which all the thermodynamic potentials are stationary. To prove this statement, we consider a small displacement $(d x, d y)=\left(r_{1}, r_{2}\right) d s$ along $\vec{r}$ from a point on the spinodal. Taking the derivative of the chemical potential $\mu_{i}$ along $\vec{r}$ we find

$$
\begin{aligned}
\frac{\partial \mu_{i}}{\partial s}= & \frac{\partial \mu_{i}}{\partial x} \frac{\partial x}{\partial s}+\frac{\partial \mu_{i}}{\partial y} \frac{\partial y}{\partial s}=\left(g_{i 1}-x g_{11}-y g_{21}\right) r_{1} \\
& +\left(g_{i 2}-x g_{12}-y g_{22}\right) r_{2}=\left(g_{i 1} r_{1}+g_{i 2} r_{2}\right) \\
& -\left(g_{11} r_{1}+g_{12} r_{2}\right) x-\left(g_{21} r_{1}+g_{22} r_{2}\right) y=0
\end{aligned}
$$

where the expressions in brackets in the last line of Eq. (16) all vanish because of Eq. (15). In general the direction $\left(r_{1}, r_{2}\right)$ will intersect with the spinodal. In special (critical) points the direction $\left(r_{1}, r_{2}\right)$ will be tangent to the spinodal. There two neighboring points will have the same thermodynamic potentials according to Eq. (16) and are thus also coexisting. In the critical points the spinodal and binodal therefore touch, and the length of the tie lines goes to zero. Critical points are hence the limiting points of coexistence.

We can use Eq. (15) to find the critical points in a ternary system. We first note that Eq. (15) implies that the second derivative of $g$ in the direction $\left(r_{1}, r_{2}\right)$ vanishes:

$$
\sum_{i, j=1}^{2} g_{i j} r_{i} r_{j}=0 \text {. }
$$

Equation (17) follows from Eq. (15) by multiplication with $r_{i}$ and summing over $i$ as well as $j$. In the critical point, where $\vec{r}=\left(r_{1}, r_{2}\right)$ is tangent to the spinodal, the determinant is stationary (remaining zero), so we have

$$
\sum_{i, j, k=1}^{2} g_{i j k} r_{i} r_{j} r_{k}=0
$$

which means that the third derivative of $g$ in the direction of $\vec{r}$ vanishes. Combined, Eqs. (17) and (18) give the conditions for a critical point.

A final question concerns the disappearance of the instability region from composition space. Then the derivative of the determinant will be zero in all directions. Equivalently, using Eq. (18) for the independent $x$ and $y$ directions, we have 


$$
g_{x x x}=g_{y y y}=0 .
$$

Together with Eq. (14), Eq. (19) determines what we will call a ternary critical point, or the onset of phase separation. Such a ternary critical point usually does not occur in a Gibbs phase triangle, but if we add an additional axis (e.g., for temperature), the resulting three-dimensional phase prism will have such a point.

\section{MODEL FOR TERNARY LIPID MIXTURES}

We denote the volume fractions of the saturated lipids, unsaturated lipids, and cholesterol by $x, y$, and $z$, respectively. Analogously to the Flory-Huggins model, we take the fully demixed state as our reference state, and consider the change in Gibbs free energy due to mixing

$$
\mathcal{G}=-T \Delta \mathcal{S}+\Delta \mathcal{G}_{\text {loc }} .
$$

The change in entropy by the increase in available volume when going from a demixed state to a mixed state is $-k_{\mathrm{B}} N_{i} \log x_{i}$ for each of the three components (where $\log$ indicates the natural logarithm, $x_{i}$ as before the number fraction of the $i$ th component and $N_{i}$ its total number of molecules). In our ternary system we have

$$
\Delta \mathcal{S}=-k_{\mathrm{B}} N[x \log x+y \log y+z \log z] .
$$

For each of the three binary mixtures we present a FloryHuggins like local energy term. We assume that the volume is extensive, i.e., scales linearly with the total number of particles $N$ in the system, and therefore $x_{i}$ is also the volume fraction of the $i$ th component. The probability for two differ-

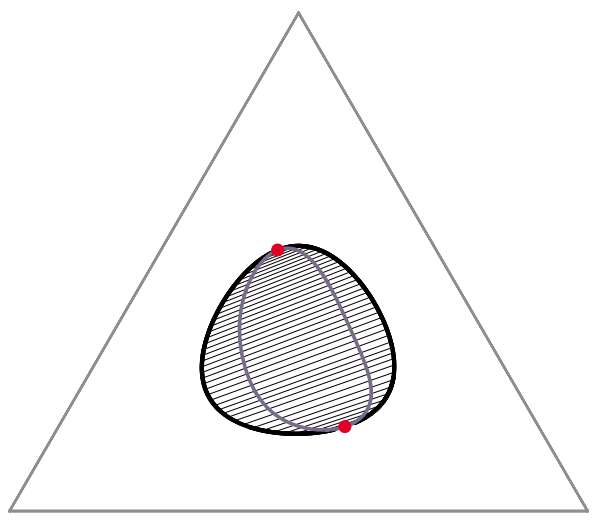

FIG. 1. (Color online) Gibbs phase triangle showing phase separation in the ternary system when there is none in any of the binary ones. The thick black line is the binodal, which marks the boundary of the immiscibility region. Any composition corresponding to a point inside the immiscibility region will result in demixing into two states, which are at the ends of the corresponding tie lines (thin black lines). The blue (gray) line inside the immiscibility region is the linear instability line (sometimes called the spinodal): points inside the region bordered by the blue line correspond to compositions that will demix by spinodal decomposition, points outside it will demix by nucleation and growth. The red (gray) dots indicate the critical points. Parameters used: $\chi_{x y}=1.5, \chi_{y z}=1.25, \chi_{x z}=0.75$, $\bar{\chi}=5.0$. ent molecules to encounter each other scales with both their volume fractions. The difference in interaction energy between two identical and two different nearest-neighbor molecules is given by the dimensionless parameter $\chi[25]$. The local interaction term for a mixture of $x$ and $y$ is therefore given by $k_{\mathrm{B}} T N \chi x y$. Below we will show that a model with just three binary interaction terms cannot reproduce the experimentally observed phase diagrams. We therefore add another term, which depends on all three volume fractions $[32,36]$. This addition supposes a significant contribution from a third-order term to the total free energy. There are two reasons why such a third-order term may occur. The first is if one of the components (here the cholesterol) acts as a line active agent for the phase separation of the other two $[39,40]$. In that case all three need to come together at a single point in space, and hence a third-order term emerges. The second option is essentially the one suggested by Radhakrishnan and McConnell [28,29], which is supported both by numerical studies [41-43] as well as some tentative experimental data $[12,44]$. It supposes that the saturated lipids and the cholesterol form complexes, which subsequently interact with the unsaturated lipids. The difference between the model of Radhakrishnan and McConnell and the one proposed here is that we simply look at the individual components, reflecting the fact that binary complexes are short-lived and continually form and dissociate, as is also seen in simulations [4]. A third-order term emerges by combining the probabilities of a two-component complex to form and it meeting up with the third component.

Combining all contributions, we postulate for the local interaction term

$$
\Delta \mathcal{G}_{\mathrm{loc}}=k_{\mathrm{B}} T N\left[\chi_{x y} x y+\chi_{x z} x z+\chi_{y z} y z+\bar{\chi} x y z\right],
$$

and for the total change in Gibbs free energy we have

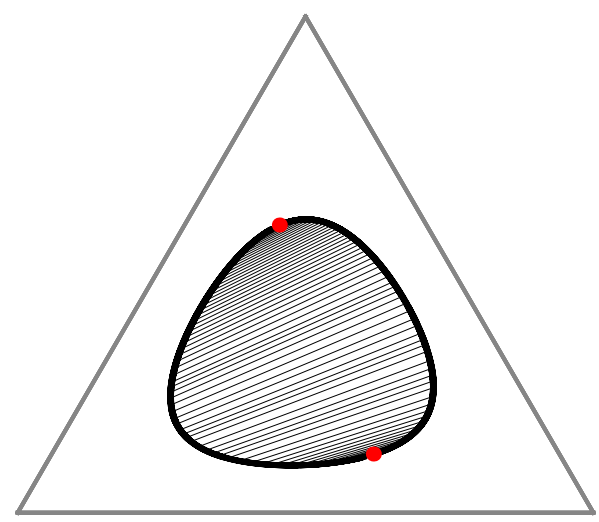

FIG. 2. (Color online) Gibbs phase triangle showing phase separation in the ternary system when there is none in any of the binary ones, but one of the binary interactions is attractive. The thick black line is the binodal, which marks the boundary of the immiscibility region. Any composition corresponding to a point inside the immiscibility region will result in demixing into two states, which are at the ends of the corresponding tie lines (thin black lines). The red (gray) dots indicate the critical points. In this case, we find numerically that the coexistence region vanishes if the value of the ternary interaction parameter $\bar{\chi}$ is set to 0 . Parameters used: $\chi_{x y}=1.5$, $\chi_{y z}=1.0, \chi_{x z}=-0.5, \bar{\chi}=5.0$. 


$$
\begin{aligned}
\frac{1}{N k_{\mathrm{B}} T} \mathcal{G}= & x \log x+y \log y+z \log z+\chi_{x y} x y+\chi_{x z} x z+\chi_{y z} y z \\
& +\bar{\chi} x y z
\end{aligned}
$$

with (as before, by definition)

$$
x+y+z=1 .
$$

Putting one of the three number fractions equal to zero in Eq. (23), we get the Flory-Huggins model for a binary system, as given by Eq. (2). A straightforward calculation which can be found in many textbooks (e.g., [25]) tells us that if the corresponding Flory-Huggins parameter $\chi$ is less than 2 the entropy term dominates and the system is in a single homogeneous phase. If $\chi>2$ a miscibility gap opens up and the free energy can be lowered by demixing into two coexisting phases.

The ternary term in Eq. (22) is the only ternary term we can add without changing the underlying binary systems, which is why we do not add any other ternary terms (e.g., an $x x y$ term). As we will show below, the ternary term is necessary to explain the existence of a closed-loop miscibility gap in systems where the interactions between any pair of the three components are repulsive (i.e., their $\chi$ parameters are positive). If there are attractive interactions instead (e.g., because one of the components is a solvent for one or both of the others), a closed-loop miscibility gap can be described in a system with just the binary interactions [32]. In that case, the closed-loop immiscibility gap results from an asymmetry in the interaction parameters between the three pairs, which is called a $\Delta \chi$ effect [34].

Substituting the free energy given by Eq. (23) in the equations of Sec. II, we can calculate Gibbs phase triangles for given values of $\chi_{x y}, \chi_{x z}, \chi_{y z}, \bar{\chi}$ and find the binodals, spinodals, and critical points. If $\chi_{x y}, \chi_{x z}$, and $\chi_{y z}$ are all less than 2 , the corresponding binary systems are homogeneous, but for $\bar{\chi}$ above a critical value the ternary system can still exhibit phase coexistence. An example of a phase diagram with such a closed-loop miscibility gap is given in Fig. 1. The figure shows the binodal and tie lines, which we determine by numerically solving the system given by Eq. (11). It also shows the spinodal [the solution of Eq. (14)], which in the model given by Eq. (23) is an algebraic expression in $x$ and $y$, and the two critical points. We find both the spinodal and the critical points by numerically solving their respective algebraic expressions. As an example of a phase diagram exhibiting a $\Delta \chi$ effect is shown in Fig. 2.

Of course, we can also set the Flory-Huggins parameter of one of the binary mixtures above its critical value 2 . If we do so with only one of them, we get a phase diagram with only one critical point, because the immiscibility region continues all the way to the edge of the Gibbs triangle (Fig. 3). In the case that two of the binary parameters allow for binary demixing, we can get more interesting phase diagrams. For certain combinations of the four parameters $\chi_{x y}, \chi_{x z}, \chi_{y z}$ and $\bar{\chi}$ there are three points in the phase triangle for which the chemical potentials match. These points are the vertices of a three-phase coexistence region. Inside there are no tie lines: any system corresponding to any of the points in the three-

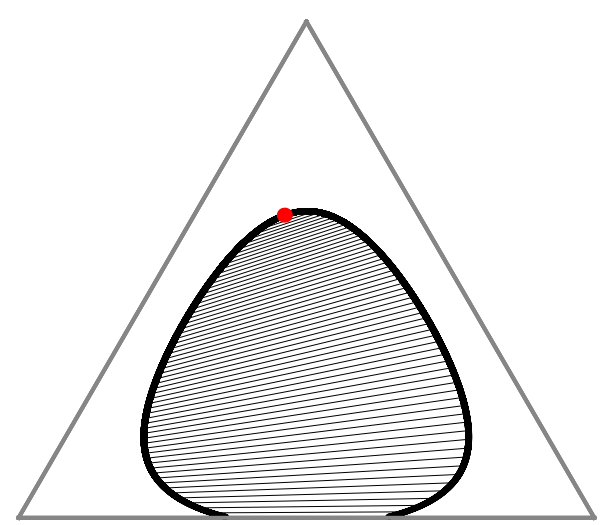

FIG. 3. (Color online) Gibbs phase triangle showing phase separation in the ternary system, when one of the underlying binary systems also exhibits phase separation. The thick black line is the binodal, which marks the boundary of the immiscibility region. Any composition corresponding to a point inside the immiscibility region will result in demixing into two states, which are at the ends of the corresponding tie lines (thin black lines). The red (gray) dot indicates the critical point. Parameters used: $\chi_{x y}=2.05, \chi_{y z}=1.25$, $\chi_{x z}=0.75, \bar{\chi}=5.0$.

phase coexistence region will demix in the same fashion. The three-phase coexistence region is bordered by three twophase coexistence regions, which we can identify as either liquid-liquid or liquid-gel by their densities. An example of such a phase diagram is shown in Fig. 4.

Finally, we use Eqs. (14) and (19) to find the conditions for having a ternary critical point. Differentiating $g(x, y)$ three times, we find (reintroducing $z$ to show the symmetry)

$$
g_{x x x}(x, y)=\frac{1}{z^{2}}-\frac{1}{x^{2}}=0,
$$



FIG. 4. (Color online) Gibbs phase triangle showing separation into two phases [the regions with the thin black and red (gray) lines, which represent tie lines] and three phases [inside the blue (gray) triangle; the compositions of the three phases correspond to the vertices of the triangle]. The regions with black tie lines correspond to the coexistence of a gel and a liquid phase; the region with the red (gray) tie lines corresponds to liquid-liquid coexistence, with a critical point indicated by the red (gray) dot. The system is in a homogeneous gel phase in the lower right-hand region and in a homogeneous liquid phase in the left-hand region. Parameters used: $\chi_{x y}=2.2, \chi_{x z}=1.95, \chi_{y z}=2.15, \bar{\chi}=4.0$. 


$$
g_{y y y}(x, y)=\frac{1}{z^{2}}-\frac{1}{y^{2}}=0 .
$$

The system consisting of Eqs. (24)-(26) has a single solution: $x=y=z=1 / 3$, which means that in our third-order theory a ternary critical point can only occur in the center of the Gibbs phase triangle. Substituting this point into Eq. (14), we find a condition on the parameters $\chi_{x y}, \chi_{x z}, \chi_{y z}$ and $\bar{\chi}$ for a ternary critical point to exist

$$
\begin{gathered}
27-6\left(\chi_{x y}+\chi_{x z}+\chi_{y z}\right)+2\left(\chi_{x y} \chi_{x z}+\chi_{x y} \chi_{y z}+\chi_{x z} \chi_{y z}\right)-\chi_{x y}^{2} \\
-\chi_{x z}^{2}-\chi_{y z}^{2}=\bar{\chi}\left(6-\frac{2}{3}\left(\chi_{x y}+\chi_{x z}+\chi_{y z}\right)-\frac{1}{3} \bar{\chi}\right) .
\end{gathered}
$$

If we do not include the third-order interaction term in Eq. (23), the right-hand side of Eq. (27) vanishes. In that case there are no solutions for $\chi_{x y}, \chi_{x z}$, and $\chi_{y z}$ all in the interval $[0,2]$. Hence a ternary critical point can only exist if at least one of the underlying binary systems either exhibits demixing (with $\chi>2$ ) or has an attractive interaction between its components $(\chi<0)$. A system with repulsive interactions between all components can therefore only exhibit a closedloop miscibility gap if $\bar{\chi}>0$. Given $\chi_{x y}(T), \chi_{x z}(T)$, and $\chi_{y z}(T)$ from the underlying binary systems, Eq. (27) gives us the critical value of $\bar{\chi}$, or equivalently the critical temperature of our ternary system.

\section{PHASE BOUNDARY AND LINE TENSION}

Invoking Van der Waals and Cahn-Hilliard theory, we can use our explicit form of the free energy Eq. (23) to calculate the energy penalty for having a phase boundary. For a detailed introduction into the scheme used here to derive an expression for the line tension, in particular Eqs. (30) and (31) for a general Gibbs free energy, see Fisk and Widom [37].

We consider two coexisting liquid phases with compositions $\left(\bar{x}_{1}, \bar{y}_{1}, \bar{z}_{1}\right)$ and $\left(\bar{x}_{2}, \bar{y}_{2}, \bar{z}_{2}\right)$, where we eliminate $z$ as usual. The concentrations do not make a jump at the domain boundary but rather have a smooth transition when we go from one domain to the other. We parametrize the "position" between the two phases by a variable $s$ : for $s \rightarrow-\infty$ we are in phase 1 and for $s \rightarrow \infty$ we are in phase 2. The origin $s=0$ is determined as the location of the Gibbs dividing surface

$$
\begin{aligned}
0= & \int_{-\infty}^{0}\left\{\lambda_{x}\left[x(s)-\bar{x}_{1}\right]+\lambda_{y}\left[y(s)-\bar{y}_{1}\right]\right\} \mathrm{d} s+\int_{0}^{\infty}\left\{\lambda_{x}\left[x(s)-\bar{x}_{2}\right]\right. \\
& \left.+\lambda_{y}\left[y(s)-\bar{y}_{2}\right]\right\} \mathrm{d} s
\end{aligned}
$$

with the constants $\lambda_{x}$ and $\lambda_{y}$ to be determined. The line tension is then given by the integral of the free-energy density $\Psi(x, y)$ (to be defined below):

$$
\begin{aligned}
\tau= & \int_{-\infty}^{0}\left\{\Psi[x(s), y(s)]-\Psi\left(\bar{x}_{1}, \bar{y}_{1}\right)\right\} \mathrm{d} s+\int_{0}^{\infty}\{\Psi[x(s), y(s)] \\
& \left.-\Psi\left(\bar{x}_{2}, \bar{y}_{2}\right)\right\} \mathrm{d} s .
\end{aligned}
$$

The key assumption of the Van der Waals and Cahn-Hilliard theory is that $\Psi$ exists for all values of $s$, and is given by the
Gibbs free energy per particle $g(x, y)$ plus a quadratic gradient that accounts for the inhomogeneity in the transition region:

$$
\Psi[x(s), y(s)]=g[x(s), y(s)]+\frac{A}{2}\left(\dot{x}^{2}+\dot{y}^{2}\right),
$$

where dots denote derivatives with respect to $s$. Here we make the simplifying assumption that the $y$-component of the "kinetic energy" term has the same "mass" $A$ as the $x$ component. We can combine the expression for the line tension with the condition Eq. (28) into a single functional, where $\lambda_{x}$ and $\lambda_{y}$ play the role of Lagrange multipliers:

$$
\begin{aligned}
\tau= & \int_{-\infty}^{\infty}\left\{g[x(s), y(s)]-\bar{g}_{12}+\frac{A}{2}\left(\dot{x}^{2}+\dot{y}^{2}\right)-\lambda_{x}\left[x(s)-\bar{x}_{12}\right]\right. \\
& \left.-\lambda_{y}\left[y(s)-\bar{y}_{12}\right]\right\} \mathrm{d} s,
\end{aligned}
$$

where $\bar{g}_{12}$ means $g\left(\bar{x}_{1}, \bar{y}_{1}\right)$ for $s \leq 0$ and $g\left(\bar{x}_{2}, \bar{y}_{2}\right)$ for $s \geq 0$ with corresponding definitions for $\bar{x}_{12}$ and $\bar{y}_{12}$. Considering the integrand of Eq. (31) as a Lagrangian, we can invoke the Euler-Lagrange equations and find that for a stable interface $(\delta \tau=0)$ we must have

$$
\begin{aligned}
& 0=A \ddot{x}-g_{x}[x(s), y(s)]+\lambda_{x}, \\
& 0=A \ddot{y}-g_{y}[x(s), y(s)]+\lambda_{y} .
\end{aligned}
$$

Because the derivatives of $x(s)$ and $y(s)$ must vanish for $s \rightarrow \pm \infty$, we find from Eqs. (32) and (33) for the values of $\lambda_{x}$ and $\lambda_{y}$ :

$$
\begin{aligned}
& \lambda_{x}=g_{x}\left(\bar{x}_{1}, \bar{y}_{1}\right)=g_{x}\left(\bar{x}_{2}, \bar{y}_{2}\right), \\
& \lambda_{y}=g_{y}\left(\bar{x}_{1}, \bar{y}_{1}\right)=g_{y}\left(\bar{x}_{2}, \bar{y}_{2}\right) .
\end{aligned}
$$

Equations (34) and (35) are identical to the first and second condition of system Eq. (12) which determines the binodal. Equations (32) and (33) are the equations giving Newton's law of motion in the $x$ and $y$ direction of a particle with mass $A$ that experiences a potential $V(x, y)$ given by

$$
V(x, y)=-g(x, y)+\lambda_{x} x+\lambda_{y} y .
$$

Moreover, since $s$ does not explicitly appear in the Lagrangian, there is a conserved quantity. In mechanics, this property corresponds to translational invariance, and the conserved quantity is equivalent to the energy of the particle system:

$$
E=\frac{A}{2}\left(\dot{x}^{2}+\dot{y}^{2}\right)+V(x, y) .
$$

Again taking the limits $s \rightarrow \pm \infty$ we find for $E$ :

$$
\begin{aligned}
E= & -g\left(\bar{x}_{1}, \bar{y}_{1}\right)+g_{x}\left(\bar{x}_{1}, \bar{y}_{1}\right) \bar{x}_{1}+g_{y}\left(\bar{x}_{1}, \bar{y}_{1}\right) \bar{y}_{1}=-g\left(\bar{x}_{2}, \bar{y}_{2}\right) \\
& +g_{x}\left(\bar{x}_{2}, \bar{y}_{2}\right) \bar{x}_{2}+g_{y}\left(\bar{x}_{2}, \bar{y}_{2}\right) \bar{y}_{2},
\end{aligned}
$$

which is identical to the third condition of Eq. (12).

So far we have expressed both $x(s)$ and $y(s)$ in $s$ independently, but in order to find an expression of the line tension as an integral over the concentration $x$, we now express $y(s)$ in $x$, and write 


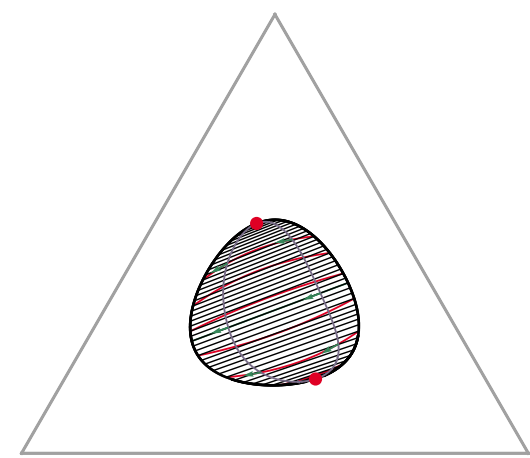

(a)

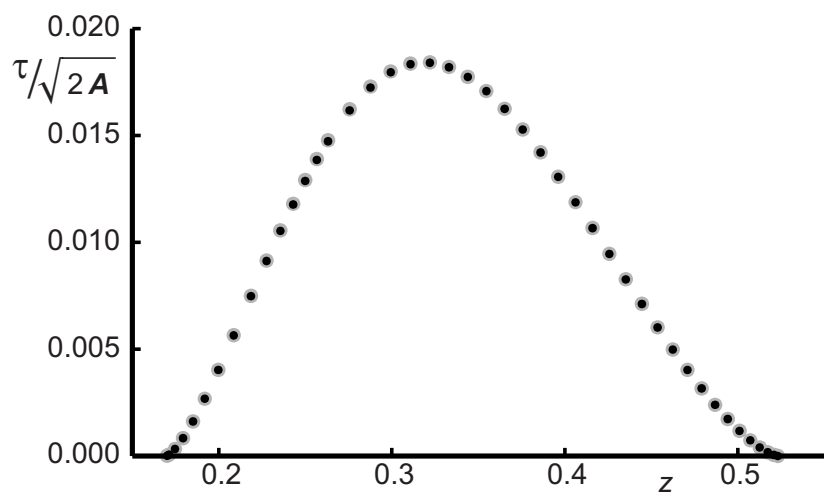

(b)

FIG. 5. (Color online) Line tension estimates using an optimized quadratic profile for $y(x)$ in Eq. (41). (a) Gibbs phase triangle showing the binodal (thick black line), tie lines (thin black lines), spinodal (blue/gray line), and critical points (red/gray dots). Some optimal quadratic paths connecting coexisting phases are shown (in red/dark gray and green/light gray), as well as the directions of the eigenvectors of the zero eigenvalues of $\left(g_{i j}\right)$ at the spinodal (in green/light gray). (b) Estimated values of $\tau / \sqrt{2 A}$ determined using the optimal quadratic profiles shown in the left figure, as a function of "position" between the critical points (the $z$ coordinate of the center of the corresponding tie line). The figure shows both the estimates determined using the optimal quadratic profiles shown in the left figure (big gray dots), as well as those determined using the optimal fourth-order profile as given in the appendix (small black dots); the positions of the points are indistinguishable in the plot. The line tension vanishes at both critical points and has a maximum when the optimal quadratic profile is a straight line, connecting the points on the binodal with the largest separation (green/light gray line in left figure). Parameters used: $\chi_{x y}=1.5, \chi_{y z}=1.25$, $\chi_{x z}=0.75, \bar{\chi}=5.0$.

$$
E=\frac{A}{2}\left[1+y^{\prime}(x)^{2}\right] \dot{x}^{2}+V[x, y(x)],
$$

where the prime denotes a derivative with respect to $x$. Equation (39) gives us an expression for $\dot{x}$ :

$$
\dot{x}=\sqrt{\frac{2}{A}} \frac{\sqrt{E-V[x, y(x)]}}{\sqrt{1+y^{\prime}(x)^{2}}} .
$$

Using Eqs. (36), (39), and (40) we can rewrite the expression for the line tension Eq. (31) as

$$
\begin{aligned}
\tau & =A \int_{-\infty}^{\infty}\left[1+y^{\prime}(x)^{2}\right] \dot{x}^{2} \mathrm{~d} s \\
& =A \int_{\bar{x}_{1}}^{\bar{x}_{2}}\left[1+y^{\prime}(x)^{2}\right] \dot{x} \mathrm{~d} x \\
& =\sqrt{2 A} \int_{\bar{x}_{1}}^{\bar{x}_{2}} \sqrt{1+y^{\prime}(x)^{2}} \sqrt{E-V[x, y(x)]} \mathrm{d} x
\end{aligned}
$$

Equation (41) again gives a functional expression for the line tension, for which we can again write down the EulerLagrange equations to get a differential equation for the optimal path $y(x)$. Because the integrand in Eq. (41) depends explicitly on $x$, there is no conserved quantity in this system. Performing the variational analysis, we find after some algebra

$$
y^{\prime \prime}(x)=\frac{1+y^{\prime}(x)^{2}}{2[E-V(x, y)]}\left[-\frac{\partial V(x, y)}{\partial y}+\frac{\partial V(x, y)}{\partial x} y^{\prime}(x)\right] .
$$

It seems straightforward to determine the optimal path from $\left(\bar{x}_{1}, \bar{y}_{1}\right)$ to $\left(\bar{x}_{2}, \bar{y}_{2}\right)$ by direct integration of the second-order differential Eq. (42). Unfortunately, there are two complications. The first is that both end points are singular points because $y^{\prime \prime}(x)$ tends to diverge close to the end points due to the factor $E-V[x, y(x)]$ in the denominator of Eq. (42). The second complication is that the integration of the entire path is highly unstable. To avoid these complications we optimize $\tau$ by making a guess for $y(x)$, and compare the guess to Eq. (42). The most obvious guess is a straight line, i.e., $y(x)$ follows the tie line that connects $\left(\bar{x}_{1}, \bar{y}_{1}\right)$ with $\left(\bar{x}_{2}, \bar{y}_{2}\right)$, which gives us an upper bound for the value of $\tau$. However, a better guess can be made by assuming a quadratic profile which has a free parameter that we can optimize [i.e., tune it such that we find the lowest possible value of $\tau$, or the best possible solution of Eq. (42)]. We notice that, according to this numerical approximation, the direction of $y(x)$ at the points at which it intersects the spinodal, coincides with that of the eigenvector $\vec{r}$ associated with the zero eigenvalue of $\left(g_{i j}\right)$, (i.e., the unstable direction, see Fig. 5). Although these quadratic profiles do not exactly solve Eq. (42), the deviation is small and only significant close to the end points. Because there the factor $\sqrt{E-V[x, y(x)]}$ in the expression for $\tau$ vanishes, the estimate for $\tau$ using the quadratic profile is a reliable one. In the appendix we show how to turn the first complication (the singular end points) into an advantage, by which we can improve the guess, using a quartic profile. However, as we also show, the improvement of the estimate of $\tau$ using this quartic profile is negligible with respect to the optimal parabola.

\section{SUMMARY AND DISCUSSION}

The model presented in this paper qualitatively describes the observed properties of the Gibbs phase triangles of ternary lipid mixtures. It reduces to the Flory-Huggins model of 
a binary system if one of the components is taken out. Moreover, we have shown that simply adding the three binary Flory-Huggins models is insufficient to reproduce certain experimentally observed features (the closed-loop miscibility gap diagram) when all binary interactions are repulsive. The physics of the ternary system is therefore more than just the sum of the physics of the constituting binary systems.

Using the ternary model we have calculated the stability properties of the various phase diagrams, and determined the stability lines or spinodals, as well as the critical points. We have also derived an expression for the line tension between two coexisting phases in a lipid membrane system, as a function of the position in the phase diagram. This approach directly couples the line tension between coexisting domains, a key factor in the determination of the shape of lipid membrane vesicles, to the composition of the membrane.

The model for the Gibbs free energy has four free parameters, of which three are obtained from the underlying binary systems and can be determined by measurements on those. The fourth parameter $(\bar{\chi})$ can be determined experimentally using, e.g., Eq. (27) for the ternary critical point. Given the values of these parameters, the value of the line tension can be calculated up to the overall proportionality factor $A$, which corresponds to a correlation length, and can in principle be determined independently.

\section{ACKNOWLEDGMENTS}

This work was supported by funds from the Netherlands Organization for Scientific Research (NWO-FOM) within the program on Material Properties of Biological Assemblies (FOM-L2601M).

\section{APPENDIX: OPTIMAL CONCENTRATION PROFILE}

Close to the binodal, the factors $\{E-V[x, y(x)]\}$, $\partial V(x, y) / \partial y$ and $\partial V(x, y) / \partial x$ in Eq. (42) all vanish. However, as we will show below, the first one vanishes quadratically with $x$, whereas the second and third only vanish linearly with $x$. Because the numerator and denominator of Eq. (42) should vanish equally fast as we approach the binodal in order for the second derivative of $y(x)$ to be well-defined, this allows us to find an expression for the first derivative of $y(x)$ at both ends of the interval. Those values we can use to improve our estimate of the concentration profile: since we now know both the end points and the derivatives at those end points, we have four set parameters and can optimize a fourth order, instead of a quadratic, profile with a single optimization parameter. We will show that the fourth-order profile gives a marginal improvement in the estimate of the line tension $\tau$, indicating that indeed the quadratic profile used in the main text gives a reliable estimate.

We rewrite Eq. (42) as an expression without fractions as

$$
\begin{aligned}
2[E-V(x, y)] y^{\prime \prime}(x)= & {\left[1+y^{\prime}(x)^{2}\right] } \\
& \times\left[-\frac{\partial V(x, y)}{\partial y}+\frac{\partial V(x, y)}{\partial x} y^{\prime}(x)\right] .
\end{aligned}
$$

We also reparameterize such that the origin is at the point around which we make our expansion [either $\left(\bar{x}_{1}, \bar{y}_{1}\right)$ or $\left.\left(\bar{x}_{2}, \bar{y}_{2}\right)\right]$. We expand $y(x)$ around this origin and write

$$
y(x)=a_{1} x+a_{2} x^{2}+a_{3} x^{3}+a_{4} x^{4}+\ldots
$$

We also define

$$
\begin{aligned}
& V_{x}=\frac{\partial V(x, y)}{\partial x}(0,0) \\
& V_{y}=\frac{\partial V(x, y)}{\partial y}(0,0)
\end{aligned}
$$

and likewise for higher-order derivatives. For the left-hand side of Eq. (A1) we then find

$$
2[E-V(x, y)] y^{\prime \prime}(x)=a_{2}\left(a_{1}^{2} V_{y y}+2 a_{1} V_{x y}+V_{x x}\right) x^{2}+O\left(x^{3}\right),
$$

where we have left out all terms which are zero by virtue of Eqs. (34), (35), and (38). The expansion of the right-hand side of Eq. (A1) gives (again leaving out terms which are zero):

$$
\begin{aligned}
{[1+} & \left.y^{\prime}(x)^{2}\right]\left[-\frac{\partial V(x, y)}{\partial y}+\frac{\partial V(x, y)}{\partial x} y^{\prime}(x)\right] \\
& =-\left(1+a_{1}^{2}\right)\left[\left(1-a_{1}^{2}\right) V_{x y}+a_{1}\left(V_{y y}-V_{x x}\right)\right] x \\
& +\frac{1}{2}\left\{-2 a_{2}\left[\left(1+5 a_{1}^{2}\right) V_{y y}+a_{1}\left(1-7 a_{1}^{2}\right)\right.\right. \\
& \left.\times V_{x y}-2\left(1+3 a_{1}^{2}\right) V_{x x}\right]+\left(1+a_{1}^{2}\right) \\
& \left.\times\left[-a_{1}^{2} V_{y y y}-a_{1}\left(2-a_{1}^{2}\right) V_{x y y}-\left(1-2 a_{1}^{2}\right) V_{x x y}+a_{1} V_{x x x}\right]\right\} x^{2} \\
& +O\left(x^{3}\right) .
\end{aligned}
$$

The lowest-order term of the left-hand side of Eq. (A1) thus goes as $x^{2}$, whereas the lowest-order term of the right-hand side goes as $x$. The coefficient of $x$ should therefore vanish for Eq. (42) to be well-defined at the binodal, which gives the condition:

$$
a_{1}^{2}-\frac{V_{y y}-V_{x x}}{V_{x y}} a_{1}-1=0,
$$

at both end points. Using condition Eq. (A7) to calculate $y^{\prime}(x)$ at $\bar{x}_{1}$ and $\bar{x}_{2}$, we have four conditions on $y(x)$. We use those to fix four of the five parameters in a fourth-order polynomial approximation of $y(x)$, leaving a single parameter which we use to optimize $\tau$ in the same fashion as we did with the quadratic approximation. Figure 5 shows the values of $\tau$ we obtain from both the quadratic and fourthorder profiles, illustrating that they are virtually the same and showing that the quadratic approximation suffices. 
[1] K. Simons and E. Ikonen, Nature 387, 569 (1997).

[2] D. A. Brown and E. London, J. Biol. Chem. 275, 17221 (2000).

[3] M. Edidin, Annu. Rev. Biophys. Biomol. Struct. 32, 257 (2003).

[4] J. F. Hancock, Nat. Rev. Mol. Cell Biol. 7, 456 (2006).

[5] F. M. Goñi, A. Alonso, L. A. Bagatolli, R. E. Brown, D. Marsh, M. Prieto, and J. L. Thewalt, Biochim. Biophys. Acta 1781, 665 (2008).

[6] C. Dietrich, L. A. Bagatolli, Z. N. Volovyk, N. L. Thompson, M. Levi, K. Jacobson, and E. Gratton, Biophys. J. 80, 1417 (2001).

[7] S. L. Veatch and S. L. Keller, Phys. Rev. Lett. 89, 268101 (2002).

[8] T. Baumgart, S. T. Hess, and W. W. Webb, Nature (London) 425, 821 (2003).

[9] R. F. M. de Almeida, A. Fedorov, and M. Prieto, Biophys. J. 85, 2406 (2003).

[10] S. L. Veatch and S. L. Keller, Biochim. Biophys. Acta 1746, 172 (2005).

[11] J. Zhao, J. Wu, F. A. Heberle, T. T. Mills, P. Klawitter, G. Huang, G. Costanza, and G. W. Feigenson, Biochim. Biophys. Acta 1768, 2764 (2007).

[12] P. F. Almeida, Biochim. Biophys. Acta 1788, 72 (2009).

[13] S. Munro, Cell 115, 377 (2003).

[14] R. Lipowsky, J. Phys. II (France) 2, 1825 (1992).

[15] A. J. Garcia-Saez, S. Chiantia, and P. Schwille, J. Biol. Chem. 282, 33537 (2007).

[16] S. Semrau, T. Idema, L. Holtzer, T. Schmidt, and C. Storm, Phys. Rev. Lett. 100, 088101 (2008).

[17] S. Semrau, T. Idema, T. Schmidt, and C. Storm, Biophys. J. 96, 4906 (2009).

[18] T. Baumgart, S. Das, W. W. Webb, and J. T. Jenkins, Biophys. J. 89, 1067 (2005).

[19] A. Tian, C. Johnson, W. Wang, and T. Baumgart, Phys. Rev. Lett. 98, 208102 (2007).

[20] A. R. Honerkamp-Smith, P. Cicuta, M. D. Collins, S. L. Veatch, M. den Nijs, M. Schick, and S. L. Keller, Biophys. J. 95, 236 (2008).

[21] P. I. Kuzmin, S. A. Akimov, Y. A. Chizmadzhev, J. Zimmerberg, and F. S. Cohen, Biophys. J. 88, 1120 (2005).

[22] S. A. Akimov, P. I. Kuzmin, J. Zimmerberg, and F. S. Cohen,
Phys. Rev. E 75, 011919 (2007).

[23] H. B. Callen, Thermodynamics and an Introduction to Thermostatistics, 2nd ed. (Wiley, New York, 1985).

[24] S. L. Veatch, K. Gawrisch, and S. L. Keller, Biophys. J. 90, 4428 (2006).

[25] G. Strobl, The Physics of Polymers, 1st ed. (Springer, Berlin, Germany, 1996).

[26] S. Komura, H. Shirotori, P. D. Olmsted, and D. Andelman, Europhys. Lett. 67, 321 (2004).

[27] S. Komura, H. Shirotori, and P. D. Olmsted, J. Phys.: Condens. Matter 17, S2951 (2005).

[28] A. Radhakrishnan and H. McConnell, Proc. Natl. Acad. Sci. U.S.A. 102, 12662 (2005).

[29] H. McConnell, Biophys. J. 88, L23 (2005).

[30] G. G. Putzel and M. Schick, Biophys. J. 95, 4756 (2008).

[31] G. G. Putzel and M. Schick, Biophys. J. 96, 4935 (2009).

[32] J. L. Meijering, Philips Res. Rep. 6, 183 (1951).

[33] J. Sivardière and J. Lajzerowicz, Phys. Rev. A 11, 2101 (1975).

[34] A. Robard, D. Patterson, and G. Delmas, Macromolecules 10, 706 (1977).

[35] M. Rabeony, D. Siano, D. Peiffer, E. Siakah-Kioulafa, and N. Hadjichristidis, Polymer 35, 1033 (1994).

[36] R. Koningsveld, W. H. Stockmayer, and E. Nies, Polymer Phase Diagrams: A Textbook (Oxford University Press, Oxford, 2001).

[37] S. Fisk and B. Widom, J. Chem. Phys. 50, 3219 (1969).

[38] P. M. Chaikin and T. C. Lubensky, Principles of Condensed Matter Physics (Cambridge University Press, Cambridge, 1995).

[39] D. J. Keller, H. M. McConnell, and V. T. Moy, J. Phys. Chem. 90, 2311 (1986).

[40] E. Sparr, K. Ekelund, J. Engblom, S. Engstrom, and H. Wennerstrom, Langmuir 15, 6950 (1999).

[41] S. A. Pandit, D. Bostick, and M. L. Berkowitz, Biophys. J. 86, 1345 (2004).

[42] S. A. Pandit, G. Khelashvili, E. Jakobsson, A. Grama, and H. L. Scott, Biophys. J. 92, 440 (2007).

[43] H. J. Risselada and S. J. Marrink, Proc. Natl. Acad. Sci. U.S.A. 105, 17367 (2008).

[44] W.-C. Hung, M.-T. Lee, F.-Y. Chen, and H. W. Huang, Biophys. J. 92, 3960 (2007). 